\title{
Utility of multidetector CT in an emergency setting in acute mesenteric ischemia
}

\author{
Barış Türkbey, Erhan Akpınar, Barbaros Çil, Muşturay Karçaaltıncaba, Okan Akhan
}

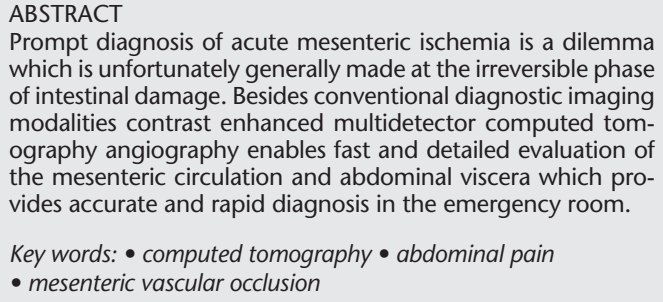

Prompt diagnosis of acute mesenteric ischemia is a dilemma which is unfortunately generally made at the irreversible phase of intestinal damage. Besides conventional diagnostic imaging modalities contrast enhanced multidetector computed tomography angiography enables fast and detailed evaluation of the mesenteric circulation and abdominal viscera which provides accurate and rapid diagnosis in the emergency room.

Key words: • computed tomography • abdominal pain

- mesenteric vascular occlusion

From the Department of Radiology (B.T. $\bowtie$ bturkbey@yahoo. com), Hacettepe University School of Medicine, Ankara, Turkey.

Received 29 February 2008; revision requested 29 February 2008; revision received 1 March 2008; accepted 3 March 2008.

Published online 5 October 2009

DOI 10.4261/1305-3825.DIR.1597-07.3
A cute mesenteric ischemia (AMI), which is a consequence of impaired blood flow to the gastrointestinal tract, is a life-threatening entity with an estimated mortality rate ranging between $60 \%$ and $90 \%(1-3)$. Among major etiologies of AMI are superior mesenteric artery (SMA) embolus, SMA thrombus, superior mesenteric vein (SMV) thrombosis and non-occlusive vasoconstriction (1). Minor causes of AMI include aortic dissection, intestinal volvulus, internal hernia, and adhesion band related extrinsic compression of the mesenteric vessels $(4,5)$. Although it is relatively rare (around 1\% of all acute abdomen referrals), prompt diagnosis and treatment of AMI is important since early management of this clinical entity increases survival (6).

\section{Diagnosis of acute mesenteric ischemia}

Diagnosis of AMI requires strong suspicion since the clinical features are usually non-specific such as abdominal pain, nausea, vomiting, or even diarrhea (7). Until late 1960s, surgical exploration or autopsy was used for diagnosis; then imaging modalities came into use as new diagnostic tools (8). Plain radiography and ultrasound are generally initial modalities used in patients presenting with acute abdomen in an emergency room, but their role is limited in the demonstration of primary and secondary signs of AMI. On plain radiographs, dilated bowel segments with air-fluid levels, pneumatosis and portal venous gas can be seen, whereas ultrasound can demonstrate mesenteric thrombus via Doppler mode but the presence of extensive gas within bowel segments and incompliance of patients may limit the accuracy of this imaging modality $(6,8,9)$.

Digital subtraction angiography (DSA) has been regarded as the gold standard imaging method in the setting of AMI. It not only enables direct visualization of the mesenteric vasculature but also provides prompt endovascular intervention opportunity (10). On the other hand, DSA is invasive, expensive, needs experience and, unfortunately, not readily available (10).

Computed tomography (CT) became a powerful tool in the management of AMI with the introduction of multidetector technology, as it provides direct visualization of the mesenteric vasculature, intestines and mesentery (7). With its high spatial resolution and volumetric 3 dimensional reconstructions, multidetector CT angiography (MDCTA) allows direct non-invasive visualization of the etiology of AMI and its insults on the intestines, and associated findings/pathologies through a great anatomical detail window $(6,11)$.

Magnetic resonance (MR) angiography is another non-invasive tool for the detection of AMI by determining mesenteric flow. It can be used as a second choice in children but its power is by far limited in the demonstration of calcified plaques $(12,13)$. 

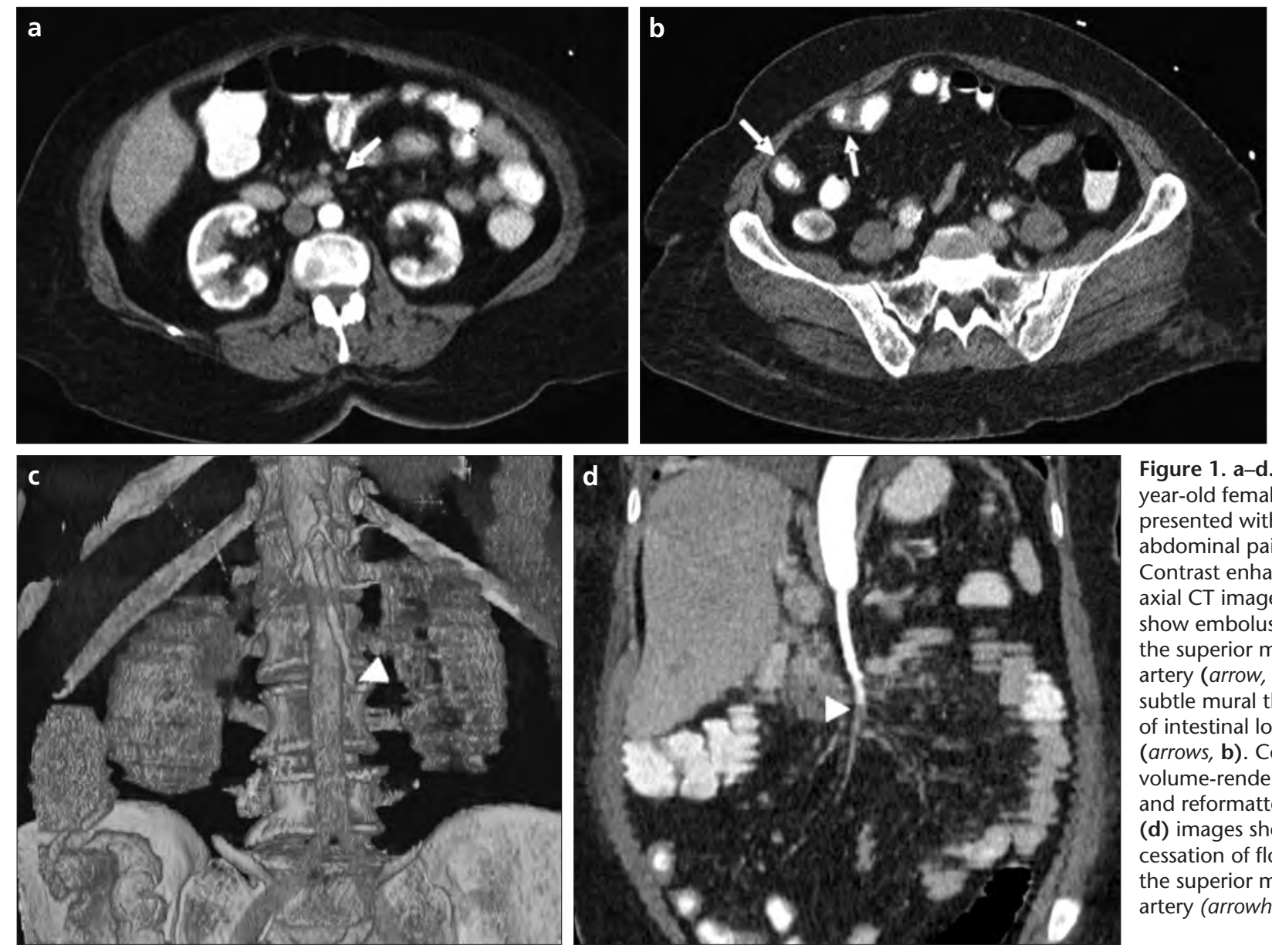

Figure 1. a-d. A 67year-old female who presented with acute abdominal pain.

Contrast enhanced axial CT images $(\mathbf{a}, \mathbf{b})$ show embolus within the superior mesenteric artery (arrow, a) and subtle mural thickening of intestinal loops (arrows, b). Coronal volume-rendered (c) and reformatted MIP (d) images show acute cessation of flow within the superior mesenteric artery (arrowheads).
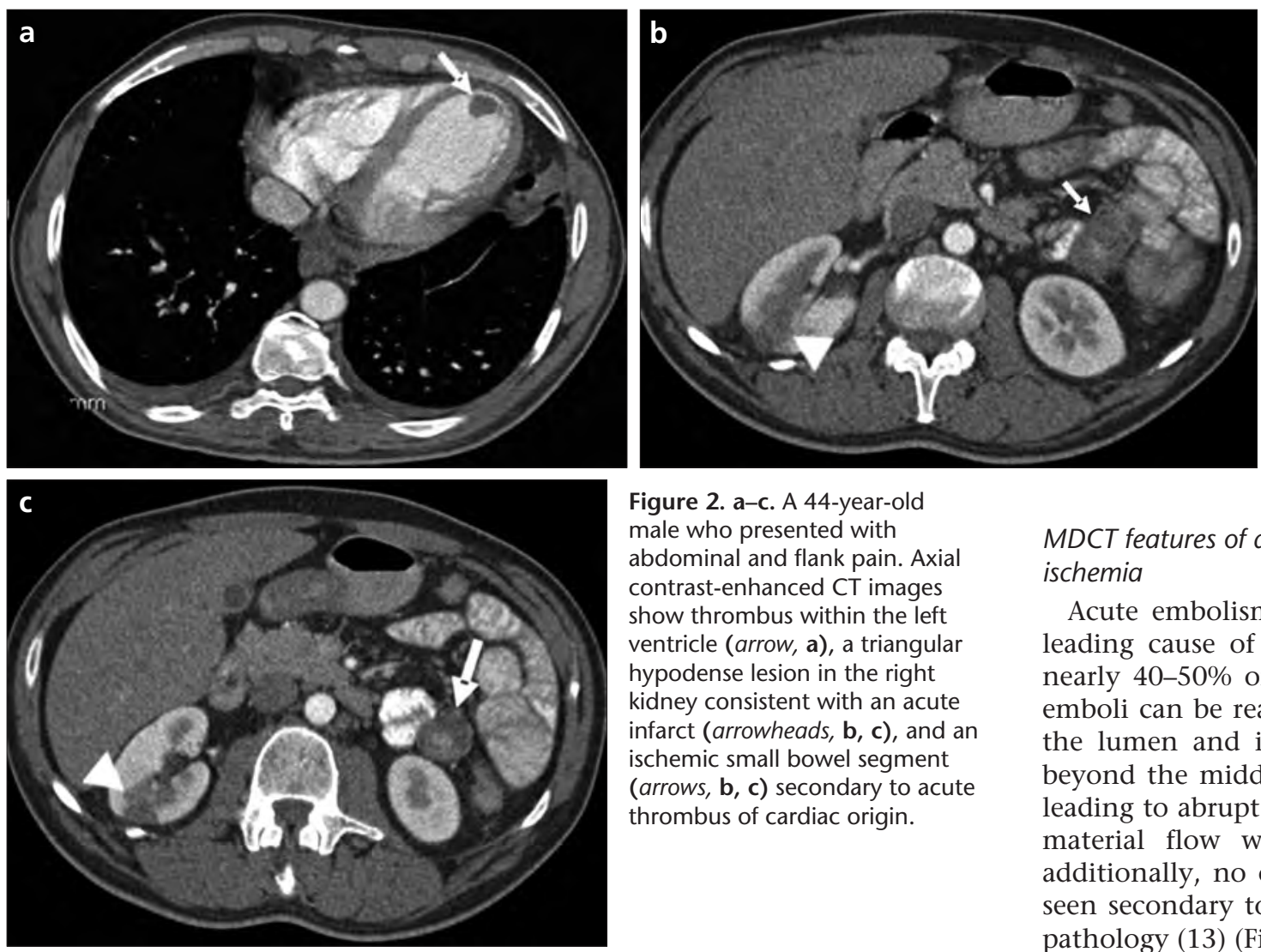

Figure 2. a-c. A 44-year-old male who presented with abdominal and flank pain. Axial contrast-enhanced CT images show thrombus within the left ventricle (arrow, a), a triangular hypodense lesion in the right kidney consistent with an acute infarct (arrowheads, b, c), and an ischemic small bowel segment (arrows, b, c) secondary to acute thrombus of cardiac origin.
MDCT features of acute mesenteric ischemia

Acute embolism in the SMA is the leading cause of AMI accounting for nearly $40-50 \%$ of all cases. Occlusive emboli can be readily detected within the lumen and is generally localized beyond the middle colic artery origin leading to abrupt cessation of contrast material flow within vessel lumen; additionally, no or few collaterals are seen secondary to acute nature of the pathology (13) (Figs. 1 and 2). 

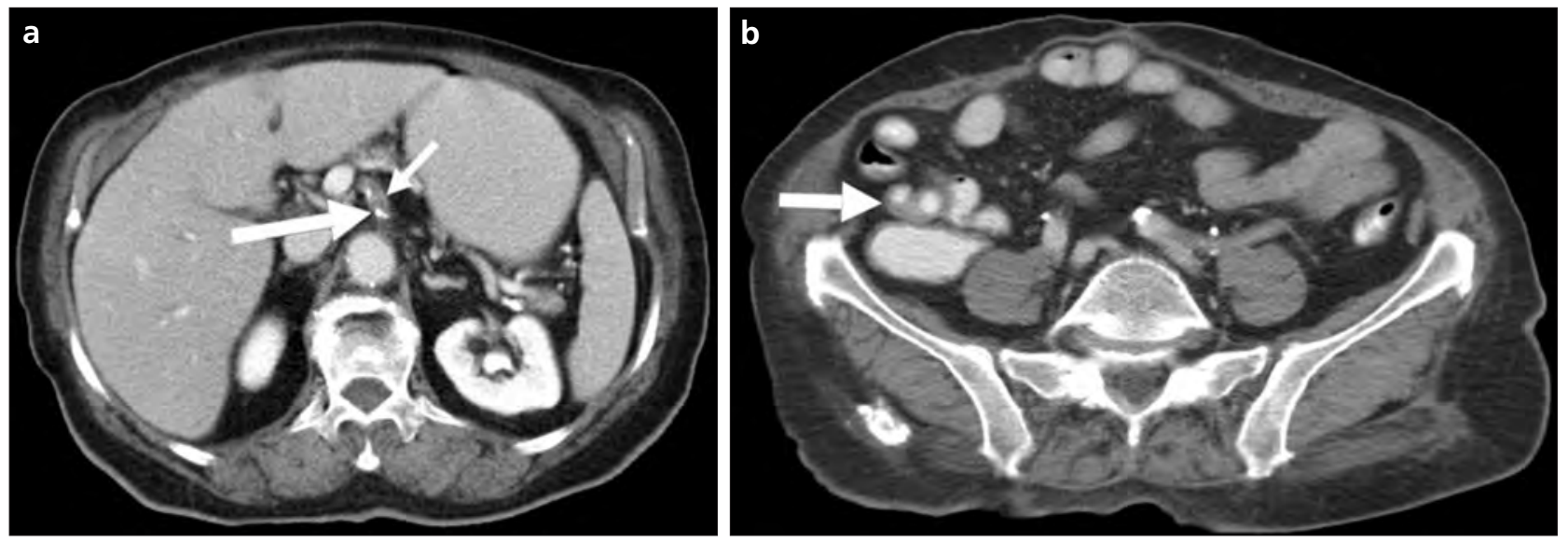

Figure 3. a, b. Axial contrast-enhanced CT images of a 77-year-old female who presented with diarrhea and acute abdominal pain. A calcified atherosclerotic lesion is seen at the wall of superior mesenteric artery (thick arrow, a). Adjacent to atherosclerotic plaques, a long segment of hypodense thrombus is seen (thin arrow, a). Moreover, subtle mural thickening (arrow, b) secondary to thrombus is seen in intestinal loops on the right.
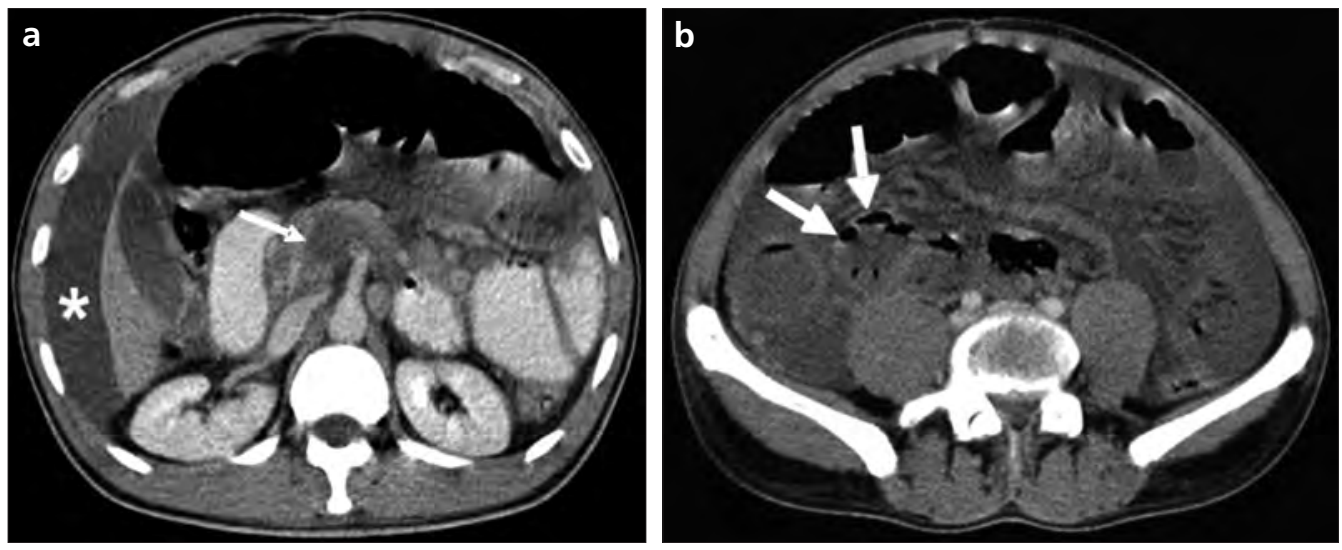

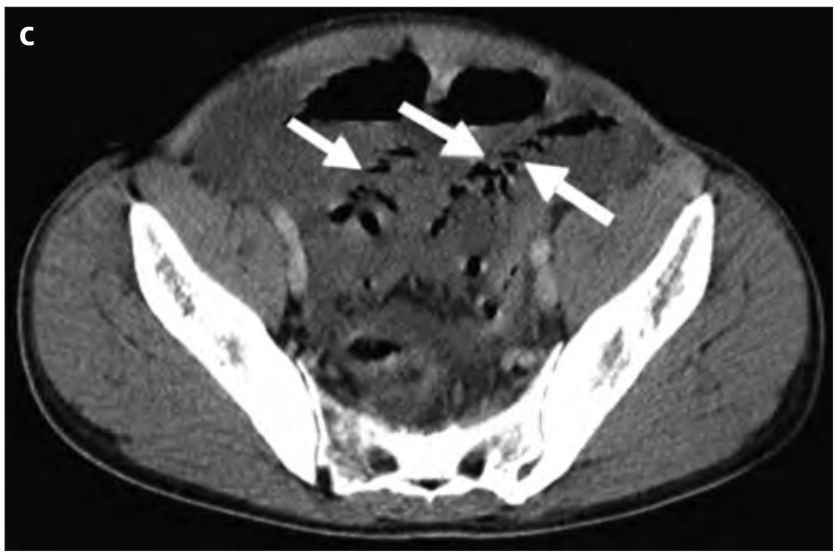

Figure 4. a-c. A 54year-old male with history of chronic liver disease who presented with diffuse abdominal pain. Axial contrast enhanced CT images show thrombus within the superior mesenteric vein (arrow, a) and ascites (asterisk, a) and pneumatosis intestinalis consistent with small bowel infarction (arrows, b and c).

In AMI secondary to mesenteric arterial thrombosis (accounting for nearly $20-30 \%$ of patients), there is almost always an accompanying preexisting atherosclerosis (13). Thrombus is generally seen relatively hypodense just adjacent to an atherosclerotic lesion, and there can be collaterals since the pathology is almost always insidious (7) (Fig. 3).

Mesenteric venous thrombosis is a rare, but potentially life-threatening, entity occurring more commonly in hypercoagulable states, trauma, portal hypertension, splenectomy, closed loop obstruction and inflammatory bowel disease $(6,14)$. It accounts for 5-15\% of AMI cases (7). Mesenteric venous thrombus is seen as a focal rounded hypoattenuating lesion with a peripheral contrast enhancement; thickness of enhancement around the venous thrombus depends on the degree of obstruction (partial or total) (7). Mesenteric venous occlusion results in edema of the intestinal mucosa which consequently results in arterial hypoperfusion if remains untreated (15) (Fig. 4).

Non-occlusive (vasoconstrictive) mesenteric ischemia is thought to be responsible for nearly $25 \%$ of AMI cases with a relatively high mortality rate (16). It develops secondary to cardiogenic shock following cardiac infarction, hypovolemia and hemorrhage, congestive heart failure, arrhythmia, aortic insufficiency, or ingestion of drugs leading to vasoconstriction such as cardiotonics and amphetamines $(17,18)$. Catheter angiography is considered as the gold standard for the diagnosis of non-occlusive mesenteric ischemia. Major angiographic findings are diffuse small mesenteric artery branches, backflow of mesenteric contrast material to the aorta, decreased flow in distal branches and decreased mesenteric venous return. However, all of these findings can be reversed via papaverine administration. Nevertheless, CT findings of this entity were defined recently in a few case reports 

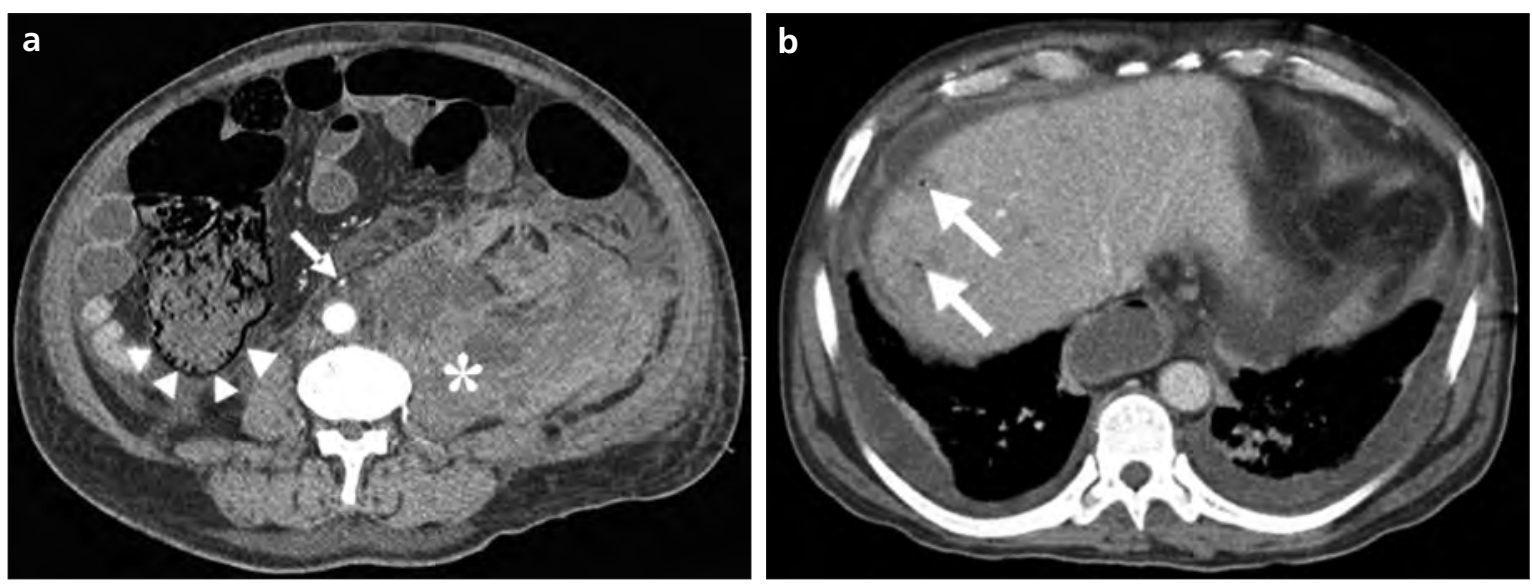
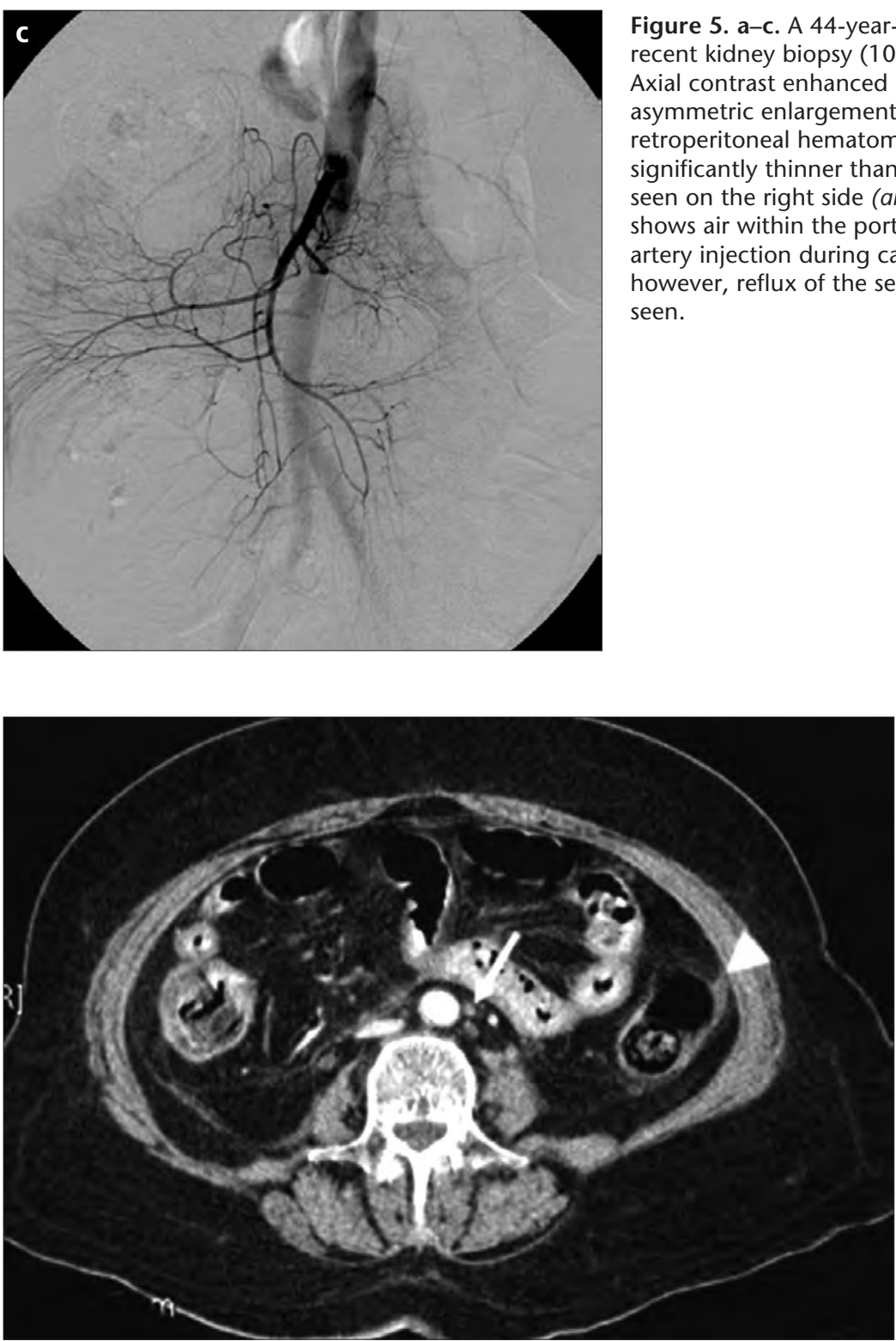

Figure 6. A 70-year-old male who presented with acute abdominal pain. Axial contrast enhanced CT image shows acute occlusion of the inferior mesenteric artery (arrow) and significant mesenteric stranding (arrowhead) adjacent to the descending colon secondary to acute ischemia.
Figure 5. a-c. A 44-year-old male with history of chronic kidney disease and recent kidney biopsy (10 days earlier) who presented with hypovolemic shock. Axial contrast enhanced abdominal CT image (a) shows significant hyperdense asymmetric enlargement of the left psoas muscle consistent with acute retroperitoneal hematoma (asterisk); the superior mesenteric artery caliber is significantly thinner than normal (arrow); moreover, pneumatosis intestinalis is seen on the right side (arrowheads). Axial CT image at the superior liver level (b) shows air within the portal vein branches (arrows). Selective superior mesenteric artery injection during catheter angiography (c) is negative for occlusive causes; however, reflux of the selectively administered contrast material to the aorta is seen. as a patent SMA trunk, diffuse small side branches that can not be visualized within distal $3 \mathrm{~cm}$ of mesentery, and absence of bowel wall thickening (19) (Fig. 5).

The most common finding of AMI regarding the intestines is mural thickening which is typically symmetric and circumferential (20) (Fig. 6). Bowel wall thickening is more commonly seen in mesenteric venous occlusion rather than in mesenteric arterial occlusion in which bowel wall can be as thin as paper due to the ischemic insult on muscle and nerve tissues (21, 22). Mural thickening can be homogenous or heterogeneous with high density areas reflecting hemorrhage accompanying hypoperfusion (23). Lack of contrast enhancement in bowel segments is a direct finding of absence of blood flow to the related bowel segment indicating infarction (24) (Fig. $7)$. Bowel dilatation is generally pronounced in the stage of irreversible transmural ischemia or infarction that interferes with peristalsis (21). Intramural gas (pneumatosis) and portal venous gas are strong indicators of infarction and worse prognosis, but they may be seen in other conditions such as over-distension, trauma, infections, neoplasm, and penetrating ulcers (25) (Fig. 8). 

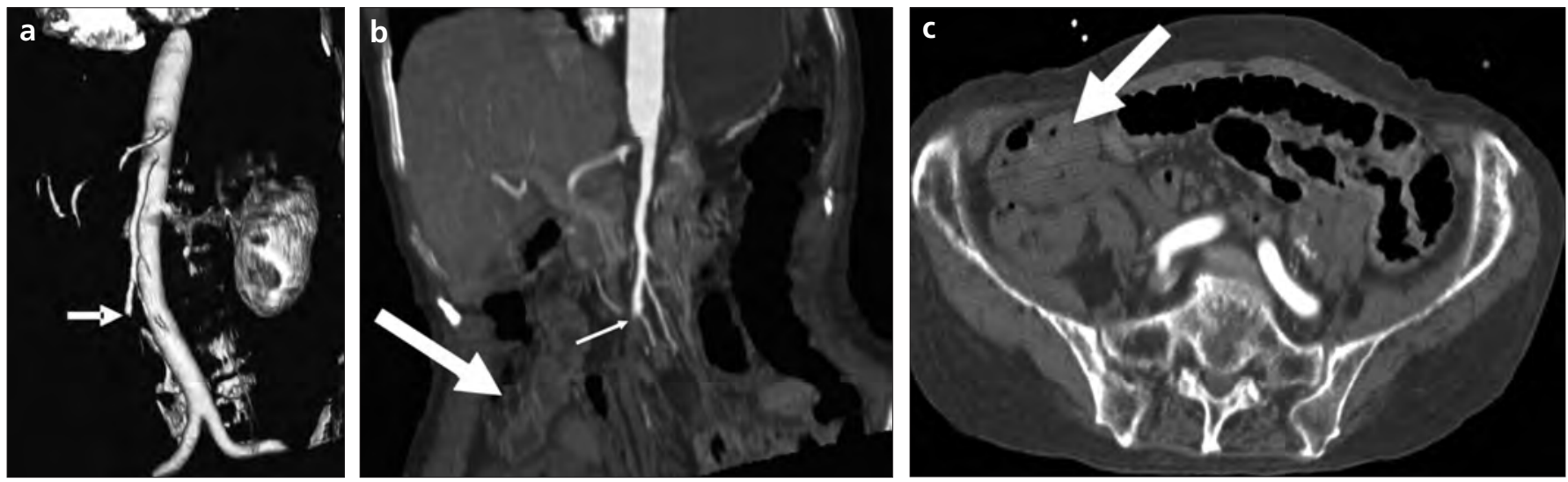

Figure 7. a-c. A 87-year-old female who presented with acute abdominal pain. Coronal oblique volume-rendered (a) and maximum intensity projection (b) CT images show acute occlusion of the distal superior mesenteric artery (arrow, a; thin arrow, b). Lack of wall enhancement consistent with infarction is seen at the level of the distal ileum and ileocecal valve on both coronal maximum intensity projection and axial CT (c) images (thick arrow, b; arrow, c).
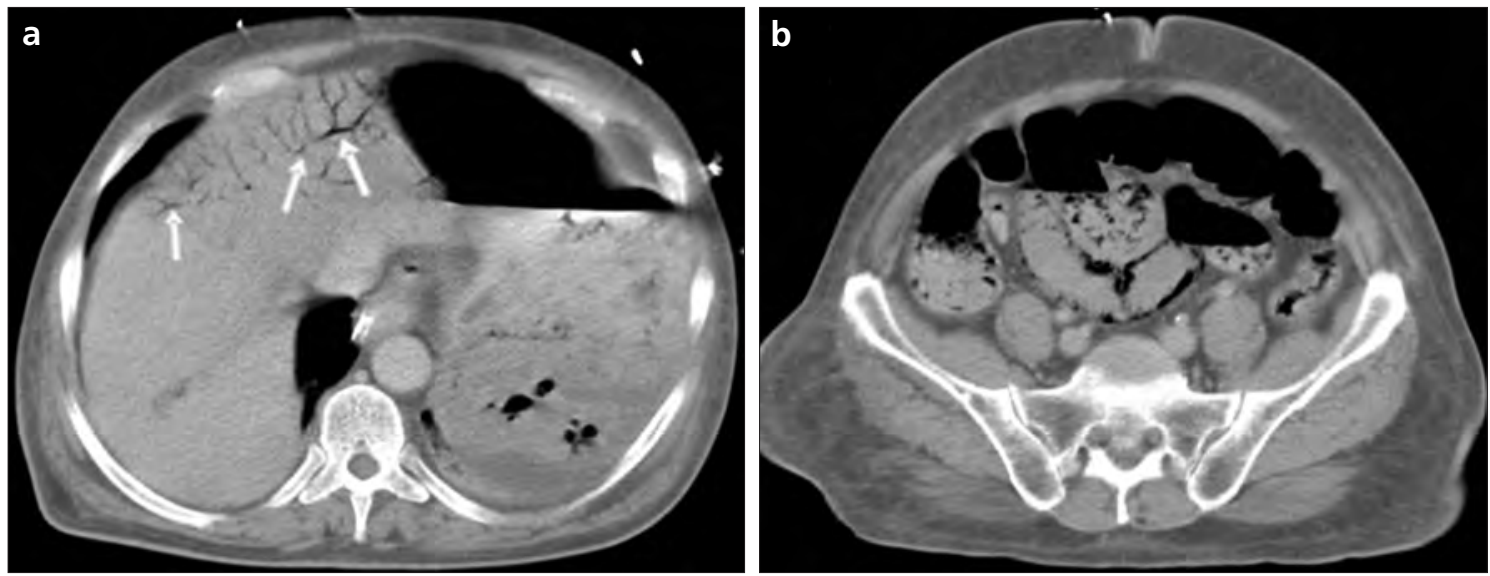

Figure 8. a, b. A 70-year-old male who presented with vague acute abdominal pain. Axial contrast enhanced CT image at lung parenchyma window setting (a) shows extensive air within branches of the left portal vein consistent with pneumoporta (arrows). Additionally, extensive air within the intestinal wall indicating pneuomatosis intestinalis (b) is seen.
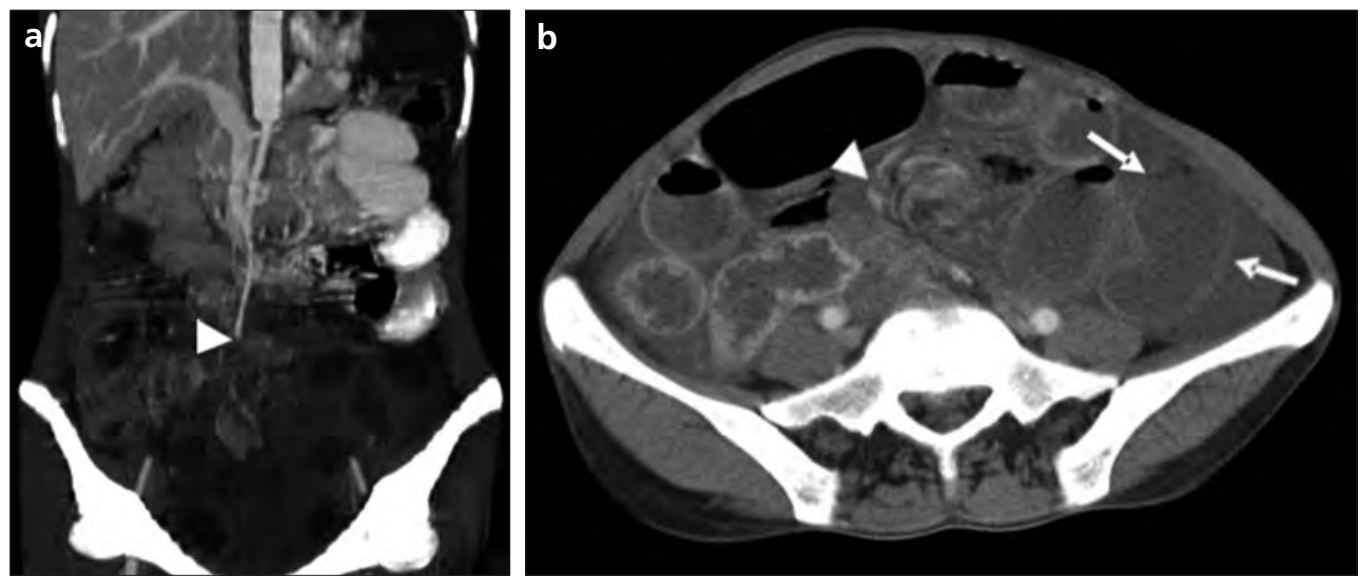

Figure 9. a, b. A 41-year-old female who presented with acute colicky abdominal pain ongoing for 4 hours. Coronal reformatted CT image (a) shows abrupt cessation of flow within the superior mesenteric artery secondary to volvulus (arrowhead). Axial contrast enhanced CT image (b) shows malrotated bowel segments on the left with a prominent perfusion defect compared to the right side (arrows). Moreover, distension of the proximal bowel segments with accompanying whirlpool sign is seen (arrowhead, b).

In case of small bowel dilatation, strangulation and ischemia should be suspected if there is ascites, diffuse mesenteric edema, mesenteric vascular engorgement, small bowel feces sign, and whirlpool sign of mesenteric vessels. These signs are regarded as ancillary CT findings of AMI, in addition to decreased mural enhancement (26) (Fig. 9). These findings, however, are nonspecific and can be seen in a wide range of acute abdomen patients such as those with pancreatitis, peritonitis, inflammatory bowel disease, perforated ulcer, and abscess (7). 


\section{References}

1. Oldenburg WA, Lau LL, Rodenberg TJ, Edmonds HJ, Burger CD. Acute mesenteric ischemia: a clinical review. Arch Intern Med 2004; 164:1054-1062.

2. Catalano O. Computed tomography in the diagnostic approach to acute mesenteric ischemia. Radiol Med 1995; 89:440-446.

3. Chabert S, Porcheron J, Balique JG. Management of acute intestinal arterial ischemia J Chir 1999; 136:130-135.

4. Lock G. Acute mesenteric ischemia: classification, evaluation and therapy. Acta Gastroenterol Belg 2002; 65:220-225.

5. Chou CK, Mak CW, Tzeng WS, Chang JM. CT of small bowel ischemia. Abdom Imaging 2004; 29:18-22.

6. Angelelli G, Scardapane A, Memeo M, Stabile Ianora AA, Rotondo A. Acute bowel ischemia: CT findings. Eur J Radiol 2004; 50:37-47.

7. Levy AD. Mesenteric ischemia. Radiol Clin North Am 2007; 45:593-599.

8. Wolf EL, Sprayregen S, Bakal CW. Radiology in intestinal ischemia. Plain film, contrast, and other imaging studies. Surg Clin North Am 1992; 72:107-124.

9. Danse EM, Van Beers BE, Goffette $P$, Dardenne AN, Laterre PF, Pringot J. Diagnosis of acute intestinal ischemia by color Doppler sonography. Color Doppler sonography and acute intestinal ischemia. Acta Gastroenterol Belg 1996; 59:140-142.

10. Bakal CW, Sprayregen S, Wolf EL. Radiology in intestinal ischemia. Angiographic diagnosis and management. Surg Clin North Am 1992; 72:125-141.
11. Hellinger JC. Evaluating mesenteric ischemia with multidetector-row CT angiography. Tech Vasc Interv Radiol 2004; 7:160-166.

12. Chow LC, Chan FP, Li KC. A comprehensive approach to MR imaging of mesenteric ischemia. Abdom Imaging 2002; 27:507-516.

13. Shih MC, Hagspiel KD. CTA and MRA in mesenteric ischemia. Part 1: role in diagnosis and differential diagnosis. AJR Am J Roentgenol 2007; 188:452-461.

14. Hatoum OA, Spinelli KS, Abu-Hajir M, Attila T, Franco J, Otterson MF, Telford GL, Binion DG. Mesenteric venous thrombosis in inflammatory bowel disease. J Clin Gastroenterol 2005; 39:27-31.

15. Wildermuth S, Leschka S, Alkadhi $\mathrm{H}_{\text {, }}$ Marincek B. Multislice CT in the pre- and postinterventional evaluation of mesenteric perfusion. Eur Radiol 2005; 15:12031210.

16. Bassiouny HS. Nonocclusive mesenteric ischemia. Surg Clin North Am 1997; 77:319-326.

17. Trompeter M, Brazda T, Remy CT, Vestring $\mathrm{T}$, Reimer P. Non-occlusive mesenteric ischemia: etiology, diagnosis, and interventional therapy. Eur Radiol 2002; 12:1179-1187.

18. Mathews JE, White RR. Primary mesenteric venous occlusive disease. Am J Surg 1971; 122:579-583.

19. Bozlar U, Turba UC, Hagspiel KD. Nonocclusive mesenteric ischemia: findings at multidetector CT angiography. J Vasc Interv Radiol 2007; 18:1331-1333.
20. Segatto E, Mortele KJ, Ji H, Wiesner W, Ros PR. Acute small bowel ischemia: CT imaging findings. Semin Ultrasound CT MR 2003; 24:364-376.

21. Horton KM, Fishman EK. Multidetector CT angiography in the diagnosis of mesenteric ischemia. Radiol Clin North Am 2007; 45:275-88.

22. Alpern MB, Glazer GM, Francis IR. Ischemic or infarcted bowel: CT findings. Radiology 1988; 166:149-152.

23. Rha SE, Ha HK, Lee $\mathrm{SH}$, et al. CT and MR imaging findings of bowel ischemia from various primary causes. Radiographics 2000; 20:29-42.

24. Zalcman M, Sy M, Donckier V, Closset J, Gansbeke DV. Helical CT signs in the diagnosis of intestinal ischemia in small-bowel obstruction. AJR Am J Roentgenol 2000; 175:1601-1607.

25. Smerud MJ, Johnson CD, Stephens DH. Diagnosis of bowel infarction: a comparison of plain films and CT scans in 23 cases. AJR Am J Roentgenol 1990; 154:99-103.

26. Balthazar EJ, Birnbaum BA, Megibow AJ, Gordon RB, Whelan CA, Hulnick DH. Closed-loop and strangulating intestinal obstruction: CT signs. Radiology 1992; 185:769-775. 